\title{
COMPARAÇÃO ENTRE JONES JIG E SLIDING JIG NO EFEITO DE INCLINAÇÃO DO MOLAR SUPERIOR OCORRIDO NA DISTALIZAÇÃO
}

\section{COMPARATIVE BETWEEN JONES AND SLIDING JIG JIG ON THE EFFECT OF INCLINATION CHANGES IN MOLAR DISTALIZATION.}

\author{
Rochely de Paula Quadros'; Elcy Arruda²; Roberley Assad²; Rodrigo Stanislawczuk ${ }^{3}$ \\ ${ }^{1}$ Rochely de Paula Quadros: Especialista em Ortodontia - Centro de Ensino Superior dos \\ Campos Gerais (CESCAGE). \\ ${ }^{2}$ Elcy Arruda: Mestre em Ortodontia -PUC-PR; Prof. do Departamento de Odontologia do \\ Centro de Ensino Superior dos Campos Gerais (CESCAGE), São Leopoldo Mandic (SLMANDIC). \\ ${ }^{2}$ Roberley Assad: Doutor em Ortodontia -SLMANDIC- Coordenador da Pós-Graduação em \\ Ortodontia do CESCAGE e SLMANDIC. \\ ${ }^{3}$ Rodrigo Stanislawczuk Grande: Mestre e Doutor em Dentística Restauradora (UEPG); \\ Prof. do Departamento de Odontologia do Centro Superior dos Campos Gerais (CESCA \\ GE), São Leopoldo Mandic (SLMANDIC). E-mail: rodrigozuk1@hotmail.com
}

Data de recebimento: $28 / 02 / 2013$

Data da aprovação: 10/05/2013

\section{RESUMO}

A presente pesquisa compara a inclinação de molares superiores durante a distalização com os aparelhos intrabucais Jones Jig e Sliding Jig. O estudo foi composto por 12 dentes, 6 dentes distalizados com o aparelho Jones Jig e 6 dentes com o Sliding Jig. Todos os dentes estavam em relação oclusal de classe II. A metodologia baseou-se em radiografias panorâmicas pré e pós-distalização, nas quais, através de linhas horizontais e verticais traçadas, quantificou-se a inclinação mésio-distal dos primeiros e segundos molares. Dos resultados coletados e estatística realizada, pode-se notar que houve diferença significativa entre os momentos iniciais e finais dos dentes distalizados com o Jones Jig e Sliding Jig, mas não foram observadas diferenças estatisticamente significantes entre os aparelhos que se mostraram semelhantes.

Palavras-chave: Distalização. Jones Jig. Sliding Jig.

\begin{abstract}
The present research compares the inclination of first and the second molar superiors during the distalization with the intrabucais appliance Jones Jig and Sliding Jig. The study it was composed for 12 teeth, 6 teeth distalized with the appliance Jones Jig and 6 teeth with the Sliding Jig. All the teeth were in class II occlusal relationship. The methodology was based in panoramic radiographs daily pay and after-distalization, where, through traced horizontal and vertical lines, it was quantified mésio-distal inclination of first and the second molar ones. Results and Conclusion of the collected results and carried through statistics, it can be noticed that it had significant difference between the initial and final moments of teeth distalized with the Jones Jig and Sliding Jig. Of the final results of the two treatments, statistical significant differences had not been observed that in summary they show that the performance of the devices was similar.
\end{abstract}

Keywords: Distalization. Jones Jig. Sliding Jig 


\section{INTRODUÇÃO}

Angle (1899) classificou as maloclusões em Classe I, II e III. A Classe II, entretanto, é a maloclusão que mais atenção recebeu devido a sua grande prevalência e pelas diversas alternativas de tratamento. Sua correção pode ser obtida através da distalização dos molares superiores, avanço mandibular em pacientes ainda em fase de crescimento ou mesmo a extração de dentes (SUGUINO et al., 2000; GONÇALVES et al., 2009). A distalização dos molares superiores no contexto da história foi empregada através do aparelho extrabucal (AEB) que apresenta como fator dependente a colaboração do paciente (HENRIQUES et al., 2009).

Necessitar desta cooperação é o que limita o uso da técnica mais tradicional e representa um problema enfrentado por muitos ortodontistas. Devido à presente variável, se faz entender desta forma o surgimento de alternativas que possam substituir o AEB. Os distalizadores intrabucais são alternativas desenvolvidas para a correção da Classe II, gerando uma força contínua nos molares superiores e ainda exigindo uma colaboração mínima ou nula do paciente (THULER et al., 2009).

Dentre os muitos distalizadores, tem-se em Jones Jig e Sliding Jig aparelhos de efeitos dentários e de bons resultados, mas que apresentam alguns efeitos colaterais, como perda da ancoragem, protrusão dos dentes anteriores e inclinação dos molares distalizados (ALMEIDA et al., 1999).

O presente estudo tem como objetivo avaliar, mediante radiografia panorâmica, o grau de inclinação dos molares após o uso dos distalizadores intrabucais Jones Jig e Sliding Jig.

\section{REVISÃO DE LITERATURA}

No início do século XX, Angle descreveu a maloclusão de Classe II como sendo uma displasia ântero-posterior caracterizada por um hipodesenvolvimento da mandíbula em relação à maxila (SUGUINO et al., 2000; LUCATO et al., 2003). Sua classificação dizia que os molares inferiores estariam em relação distal com os primeiros molares superiores (CAPELOZZA 2004). A Classe II é muito rica em características e também apresenta várias formas de tratamento (CAPELOZZA 2004). Com etiologia multifatorial, sendo esquelética ou dentária (SUGUINO et al., 2000; OLIVEIRA et al., 2004) e embora ela possa mostrar alterações verticais e um estreitamento transversal da maxila, as displasias mais frequentes da maloclusão de Classe II ocorrem no plano sagital (GONÇALVES 2009; VARGERVIK; HARVOLD, 1985). Sendo assim, a distalização dos molares superiores é um protocolo usual na Ortodontia, e o uso de aparelhos distalizadores surgiu há mais de dois séculos, com Cellier em 1802 (FERREIRA, 2008).

O diagnóstico da maloclusão de Classe II é de suma importância para a elaboração de um plano de tratamento, assim como selecionar a melhor mecânica a ser empregada para a sua correção. Diagnosticar uma maloclusão de Classe II esquelética ainda em fase de crescimento requer a utilização de aparelhos ortopédicos ou mecânicos que farão a correção ântero-posterior melhorando o perfil facial e minimizando as chances de extrações dentárias. Já na Classes II relacionada a fatores dentários, associada à protrusão da maxila tornam-se necessárias as extrações dentárias ou a distalização dos molares superiores (ALMEIDA et al., 1999).

O tratamento da maloclusão de Classe II podese dar com a distalização dos molares superiores através de recursos extras ou intrabucais (OLIVEIRA et al., 2004). Umas das técnicas mais tradicionais é a utilização do AEB, cuja grande desvantagem é depender inteiramente da colaboração do paciente (PATEL et al., 2009). Ocorre uma preferência por tratamentos que necessitem o menos possível da cooperação do paciente, de forma a ser possível ter maior controle do tratamento e produzir melhores resultados (BOLLA et al., 2002). Os distalizadores intrabucais surgiram para resolver o inconveniente da cooperação e tem se tornado frequente na Ortodontia. No entanto, a literatura sustenta que, por ser o único aparelho capaz de evitar a perda de ancoragem, controlar o centro de rotação e por ter suas forças reacionais fora da cavidade bucal, gerando movimento de translação dos dentes, o AEB parece ser superior aos aparelhos intrabucais (SILVA FILHO et al., 2006; 2007).

A opção pelo distalizador a ser utilizado deve levar em consideração a praticidade, custo, eficácia e, acima de tudo, os efeitos colaterais decorrentes de seu uso (MAIA et al., 2004). Dentre os muitos distalizadores que foram surgindo têm-se os sistemas de forças que podem ser: fios, cursores com elásticos, 
Herbst, Jasper Jampers, arco de Wilson, Ertty System, magnetos, pendulum, distal jet, o Jones Jig, entre outros.

Um método utilizado para a distalização de molares é o Sliding Jig associado à mecânica de elásticos intermaxilares que servem de ancoragem na arcada oposta (ALMEIDA et al., 1999; GONCALVEZ et al., 20010). Outro distalizador intrabucal é o Jones Jig, preconizado por Jones e White em 1992 como sendo mais uma opção para a distalização de molares (SUGINO et al., 2000; MAIA et al., 2004). Suas indicações são para casos de Classe II dentária devido à rotação dos molares, Classe III esquelética associada à Classe II dentária em que o paciente apresenta um perfil côncavo proporcionado por retrusão maxilar, pacientes que recusem o tratamento com AEB, Classe II unilateral ou bilateral, padrão mésio ou braquicefálico e pacientes com padrão facial Classse I e Classe II dentária (OLIVEIRA et al., 2004; HENRIQUES et al., 2005).

O Jones Jig libera forças suaves e contínuas, é um aparelho de fácil instalação e baixo custo que promove uma distalização rápida e satisfatória (MAIA et al., 2004). No entanto, como desvantagem, está a necessidade de vários procedimentos clínicos para sua instalação, a necessidade de bandas nos pré-molares e a impossibilidade de controlar o centro de rotação dos molares, permitindo a perda ancoragem e a inclinação dos incisivos superiores, além de aumento no transpasse horizontal e altura facial ântero-inferior e rotação dos molares (OLIVEIRA et al., 2004; PATEL et al., 2009). Ainda tem o movimento distal que não ocorre de corpo e assim se torna evidente a inclinação distal da coroa. O movimento de translação não ocorre porque o vetor de força não passa pelo centro de resistência que se encontra próxima de trifurcação das raízes (SUGUINO et al., 2000).

Outro método utilizado para a distalização de molares é o Sliding Jig que foi descrito por Tweed. Esta mecânica utiliza um cursor deslizante tracionado por elásticos intermaxilares que servem de ancoragem na arcada oposta (LUCATO et al., 2003; ALVES et al., 2005). Os cursores deslizantes apresentam baixo custo, fácil confecção, não causam desconforto ao paciente, sendo uma mecânica que pode ser utilizada tanto no arco superior como no arco inferior para obtenção de uma relação molar de Classe I (ALVES et al., 2005; SANTOS et al., 2007). Os cursores desli- zantes apresentam muitas funções, como a correção da maloclusão de Classe II por meio da distalização dos molares superiores, correção da Classe III através da verticalização dos molares inferiores, retração de pré-molares e caninos após o uso dos distalizadores, ancoragem e recuperação do perímetro do arco.

Assim a mecânica do Sliding Jig nada mais é que o uso de cursores deslizantes associados com elásticos intermaxilares de Classe II ou Classe III, necessitando de um preparo de ancoragem no arco oposto. É considerada uma alternativa viável que apresenta muitas vantagens, uma mecanoterapia fácil e de baixo custo, pois dispensa fase laboratorial. É simples e eficiente em movimentar os molares, é de rápido manuseio, com boa aceitação por parte do paciente, mas que requer colaboração. Portanto, mesmo dadas as mais diferentes formas de distalização, o aparelho de escolha deve ser aquele cuja construção e instalação o profissional domine e conheça a sua ação e principalmente os efeitos colaterais, assim como seja confortável ao paciente e corrija a maloclusão presente (MOSCARDINI et al., 200; LUCATO, et al., 2003).

As maloclusões de Classe II são as que mais receberam atenção dos estudiosos da Ortodontia, talvez porque é a mais incidente em todas as etnias estudadas (CAPELOZZA, 2004). A prevalência desta maloclusão atinge em média $60 \%$ da população ortodôntica (MANHÃES et al., 2009).

\section{MATERIAL E MÉTODOS}

A amostra selecionada foi composta de 12 molares superiores divididos em 2 grupos: grupo 1 com 6 dentes distalizados com Jones Jig e grupo 2 com 6 dentes distalizados com o aparelho Sliding Jig. A amostra foi tratada no curso de pós-graduação em Ortodontia do CESCAGE- Centro de Ensino Superior dos Campos Gerais. A inclinação mésiodistal dos molares superiores permanentes foi avaliada por meio de radiografias panorâmicas, tomadas no início e no fim da distalização, medindose o ângulo formado através de linhas verticais e horizontais. O planejamento ortodôntico incluiu a mecânica de distalização para corrigir a Classe II e levar a uma relação de Classe I. A distalização foi encerrada quando os molares distalizados estavam sobrecorrigidos e os pacientes encaminhados para uma nova radiografia panorâmica pós-distalização. 
Os molares distalizados com o Jones Jig (Figura 1 e 2) tiveram como ancoragem o botão de Nance modificado, com bandas nos segundos prémolares. Após instalação do aparelho de ancoragem e confeccionada as bandas nos primeiros molares superiores, instalou-se o Jones Jig, ativando-se a mola de NITI com fio de amarrilho 0.25 ". As ativações foram realizadas a cada 2 meses até sobrecorregir a Classe I.

A ancoragem do grupo do Sliding Jig (Figura 3) foi feita através do uso dos elásticos intermaxilares ao mesmo tempo que os elásticos ancorados no Sliding Jig ao molar inferior realizavam a distalização dos molares superiores.

Mediante a radiografia panorâmica quantificou-se a inclinação mésio-distal do longo eixo dos primeiros e segundos molares superiores e, assim, compararam-se os resultados iniciais e finais. Através de uma linha horizontal passando pelo plano palatino posterior (ENP-ENP) e linhas verticais passando pelo longo eixo dos primeiros e segundos molares, avaliou-se o ângulo formado pelas linhas verticais e horizontais (Figura 4).

Figura 1 - Aparelho Jones Jig composto por 3 estruturas, um corpo principal, mola de NITI de secção aberta e um cursor

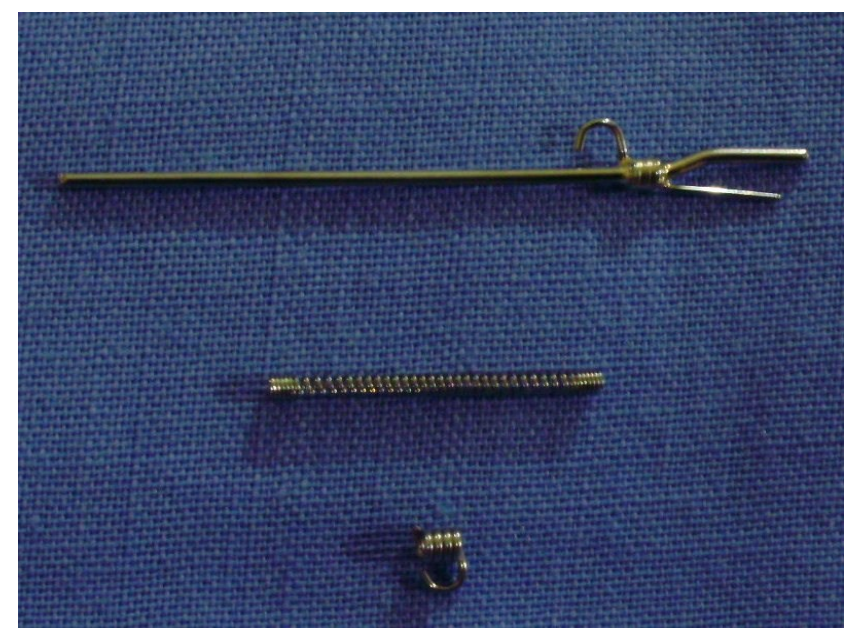

Figura 2 - Aparelho Jones Jig

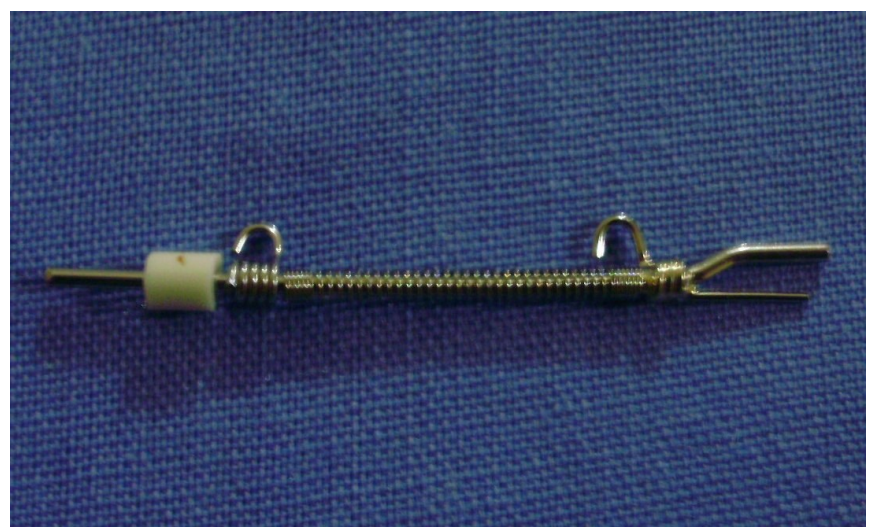

Figura 3 - Sliding Jig

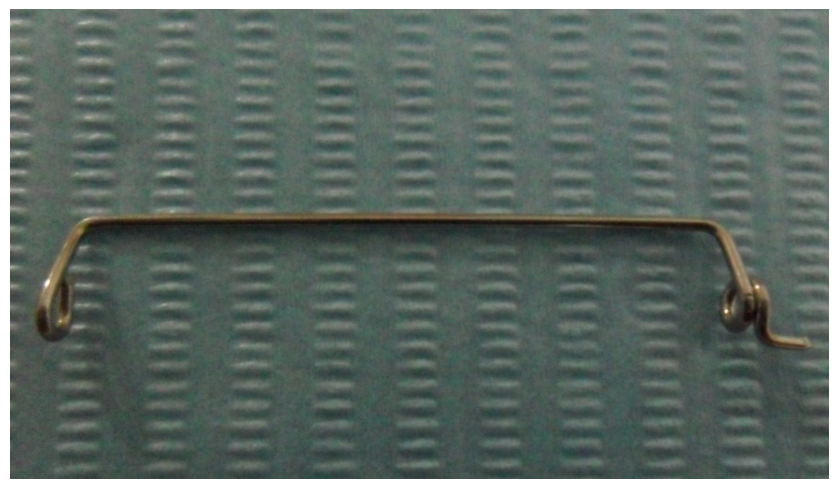

Figura 4 - Radiografia panorâmica demonstrando a metodologia empregada no estudo, através de uma linha horizontal a qual representa o plano palatino e linhas verticais representando o longo eixo dos dentes. O ângulo formado por estas linhas representa a inclinação mésiodistal dos dentes distalizados

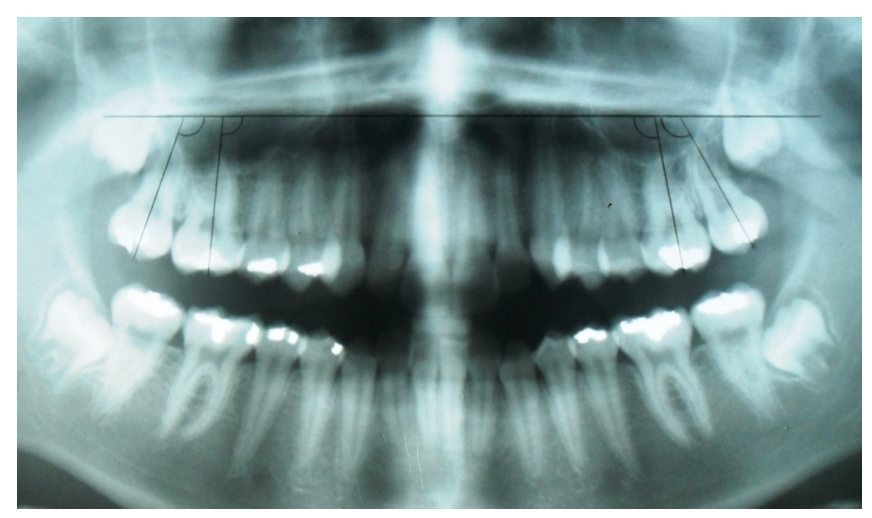




\section{RESULTADOS}

Os resultados descritos foram obtidos utilizando-se o teste estatístico paramétrico $t$ Student para amostras pareadas (comparação intragrupo) e não pareadas (comparação intergrupos), com nível de significância de 5\%. Cálculos foram realizados utilizando-se o programa Biostat 5.0. Os valores iniciais da inclinação dental e da alteração sagital sofrida pós-tratamento podem ser verificados na Tabela 1 e 2 .

Inicialmente, os dados foram testados em relação a sua normalidade, utilizando-se o teste de Kolmogorov-Smirnov. Todos os grupos passaram no teste de normalidade $(\mathrm{p}<0,10)$, assim como se observou ausência de diferença significativa entre os desvios padrão da amostra $(\mathrm{p}=0,5978)$.

A comparação entre as angulações iniciais dos molares de ambos os grupos foi realizada, utilizandose o teste $t$ Student para amostras não pareadas e não foi encontrada diferença estatísticamente significativa $(p=0,1274)$. Este resultado demonstra homogeneidade amostral no início do tratamento, ou seja, em ambos os grupos as condições pré-tratamento eram semelhantes.

Para o grupo tratado com Jones Jig, houve diferença significativa entre os momentos iniciais e finais $(p=0,0479)$, o mesmo ocorrendo com o grupo que utilizou o Sliding Jig $(\mathrm{p}=0,0182)$. (Gráfico 1)

Todavia, ao se compararem os resultados finais dos dois tratamentos, não são observadas diferenças estatisticamente significativas $(p=0,3567)$. (Grafico 1$)$

Os resultados em valores mostraram que o aparelho Sliding Jig promoveu maior inclinação do molar, porém a análise estatística de dados demonstra que não houve diferenças estatisticamente significantes.

Tabela 1 - Valores individuais (em graus) iniciais e finais da inclinação de primeiros e segundos molares distalizados com Jones Jig e Sliding Jig *

\begin{tabular}{c|c|c|c|c}
\hline & $\begin{array}{c}\text { JONES JIG - } \\
\text { inicial }\end{array}$ & $\begin{array}{c}\text { JONES JIG }- \\
\text { final }\end{array}$ & $\begin{array}{c}\text { SLIDING } \\
\text { JIG - inicial }\end{array}$ & $\begin{array}{c}\text { SLIDING } \\
\text { JIG }- \text { final }\end{array}$ \\
\hline 1 & $104,5 \mathrm{a}$ & $108,5 \mathrm{~b}$ & $107 \mathrm{a}$ & $112,5 \mathrm{~b}$ \\
\hline 2 & $109 \mathrm{a}$ & $118 \mathrm{~b}$ & $102 \mathrm{a}$ & $109 \mathrm{~b}$ \\
\hline 3 & $109 \mathrm{a}$ & $114,5 \mathrm{~b}$ & $99 \mathrm{a}$ & $113 \mathrm{~b}$ \\
\hline 4 & $105 \mathrm{a}$ & $113 \mathrm{~b}$ & $106 \mathrm{a}$ & $134 \mathrm{~b}$ \\
\hline 5 & $117 \mathrm{a}$ & $124 \mathrm{~b}$ & $116 \mathrm{a}$ & $122 \mathrm{~b}$ \\
\hline 6 & $121 \mathrm{a}$ & $115 \mathrm{~b}$ & $109 \mathrm{a}$ & $112,5 \mathrm{~b}$ \\
\hline
\end{tabular}

*letras iguais significam ausência de diferenças estatisticamente significativas
Tabela 2 - Valores individuais (em graus) média, desvio padrão, valores máximos e mínimos iniciais e finais da inclinação dos molares distalizados com Jones Jig e Sliding Jig *

\begin{tabular}{c|c|c|c|c}
\hline & $\begin{array}{c}\text { JONES JIG } \\
\text { - inicial }\end{array}$ & $\begin{array}{c}\text { JONES JIG } \\
- \text { final }\end{array}$ & $\begin{array}{c}\text { SLIDING } \\
\text { JIG - inicial }\end{array}$ & $\begin{array}{c}\text { SLIDING } \\
\text { JIG - final }\end{array}$ \\
\hline MÉDIA & 110,9 & 115,5 & 106,5 & 117,2 \\
\hline $\begin{array}{c}\text { DESVIO } \\
\text { PADRÃO }\end{array}$ & 6,7 & 5,2 & 5,9 & 9,3 \\
\hline MÁXIMO & 121 & 124 & 116 & 134 \\
\hline MÍNIMO & 104,5 & 108,5 & 99 & 109 \\
\hline
\end{tabular}

Gráfico 1 - Médias das angulações iniciais e finais de molares tratados com Jones Jig ou Sliding Jig. Comparação inter-grupo: inicial - $\mathrm{p}=0,1052$; final $-p=0,3567$. Teste t de Student para amostras não pareadas. Comparação intra-grupo: Jones Jig $-\mathrm{p}=0,0479$; Sliding Jig $-\mathrm{p}=0,0182$. Teste $\mathrm{t}$ de Student para amostras pareadas

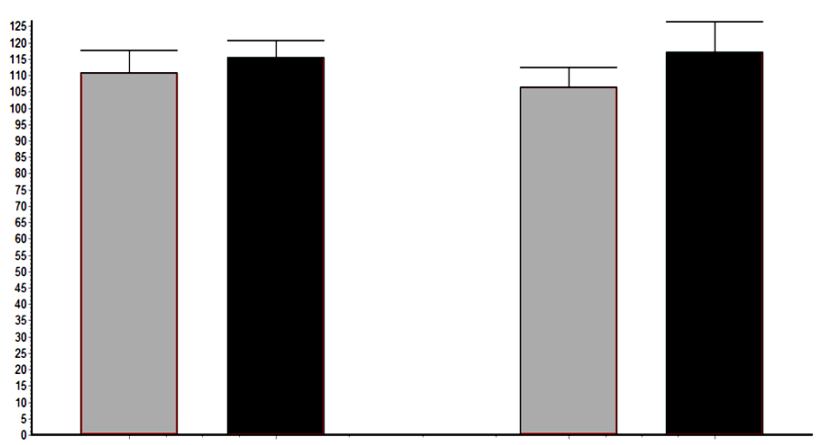

\section{DISCUSSÃO}

Mesmo a literatura confirmando o sucesso no emprego do AEB, o seu uso, muitas vezes, não é aceitável pelo paciente por questão de estética e tempo de utilização, indicando-se assim os aparelhos distalizadores intrabucais. Suguino e colaboradores (2000) quando indicam o aparelho Jones Jig recomendam que o tubo da banda seja posicionado o mais próximo do centro de resistência, para que o movimento seja de translação. Almeida e colaboradores (1999) não questionam a posição do tubo da banda, mas citam que o movimento de corpo dos molares é diretamente proporcional à quantidade de força aplicada e suas ativações.

Oliveira et al. (2004) analisaram a inclinação distal tanto dos primeiros molares como dos segundos 
molares e observaram maior movimento pendular que o de translação. Maia et al. (2004) também encontraram resultados como vestibularização e maior perda de ancoragem com o uso do Jones Jig, concluindo que o aparelho apresenta mais efeitos dentários que esqueléticos.

No tratamento com o Sliding Jig recomendase cuidado com a componente de extrusão quando se utiliza a mecânica de elásticos, principalmente em pacientes verticais (LUCATO et al., 2004). O uso dos elásticos de classe II pode diminuir ou eliminar a perda de ancoragem nas distalizações (KARLSSON et al., 2006). Nosso trabalho observou que os valores das angulações iniciais e finais obtidas indicaram que houve inclinação distal nos primeiros e segundos molares distalizados. Oliveira e colaboradores (2004) afirmaram em estudo que o Jones Jig inclina mais os molares do que os distaliza.

A inclinação distal dos molares pode ser avaliada por diversos métodos, como radiografias panorâmicas, telerradiografia em norma lateral e modelos de gesso (MAVROPOULUS, et al., 2006). Esta trilogia promove um monitoramento abrangente da distalização dos molares superiores além de avaliar a perda de ancoragem (SILVA et al., 2007). No presente trabalho utilizou-se somente a radiografia panorâmica para a verificação da inclinação distal dos primeiros e segundos molares. Baseado na metodologia de Silva Filho et al. (2007), quantificouse o grau de inclinação através de linhas traçadas na horizontal e vertical, medindo-se o ângulo entre estas linhas. Na elaboração deste método, pode-se citar a praticidade na obtenção dos resultados. No entanto, o exame radiográfico deve estar nítido, sem distorções, as linhas devem ser corretamente traçadas para que os resultados sejam equivalentes ao real movimento.

Em se tratando de colaboração, o Jones Jig não depende da colaboração do paciente (HENRIQUES et al., 2005), ao contrário do Sliding Jig que é uma mecânica que utiliza elásticos intrabucais e o paciente deve fazer o uso e a troca dos elásticos constantemente para que ocorra o movimento desejado (LUCATO et al., 2004). No presente trabalho notou-se cooperação do paciente nos dentes distalizados com o Sliding Jig. A aceitação observada pode-se atribuir ao fato de que os elásticos sejam intrabucais; em contrapartida o AEB, por ser extrabucal, ocasiona uma rejeição de uso por parte do paciente devido à estética.
Com os valores obtidos, pode-se perceber que tanto os dentes distalizados com o Jones Jig como com o Sliding Jig apresentaram inclinação distal e com os valores médios nota-se que a maior inclinação foi com o aparelho Sliding Jig. Porém quando se aplicou o Teste $t$ Student nenhuma diferença estatística foi encontrada.

Oliveira et al. (2004) obtiveram $8.2^{\circ}$ de inclinação para os primeiros molares e $9,1^{\circ}$ para os segundos. Maia et al. (2004)) também verificaram que o Jones Jig causa maior movimento pendular, não promovendo assim distalização efetiva de raiz. Em linhas gerais, ambos os molares acabaram inclinando, porém os segundos em comparação ao primeiro apresentaram maior inclinação, discordando dos resultados do presente estudo, onde se verificou que, com os valores das angulações obtidas, o Jones Jig teve menor inclinação. Os possíveis motivos desta diferença podem estar associados à força dissipada pelo Jones Jig, através da mola de níquel titânio com liberação de forças suaves e contínuas que a cada consulta decrescem.

A preocupação de Lucato et al. (2004) no tratamento do Sliding Jig é com a componente de extrusão e giro do molar. Moscardini et al. (2010) também salienta a importância no controle da mecânica de elásticos com cursor através de dobras nos arcos ortodônticos para evitar os efeitos colaterais. Na literatura revisada não foram encontrados estudos mais específicos sobre o movimento de inclinação do molar com o uso do Sliding Jig. Portanto não há como afirmar o que influenciou para que os valores dos molares distalizados com o Sliding Jig se apresentassem com maior inclinação.

Em 2004, Kinzinger et al. verificaram que a inclinação dos primeiros molares é maior quando o segundo molar ainda está em fase de erupção, e que os segundos também inclinam quando o terceiro está na direção do movimento. Esse estudo avaliou a hipótese sobre a distalização dos primeiros molares em relação à posição dos segundos e terceiros molares. Baseado em avaliação cefalométrica sagital analisou-se que na direção da distalização o germe do dente do segundo molar atua na mesial de dente como um fulcro, pois serve de resistência distal, mas ambos acabam sendo distalizados.

A distalização dos primeiros molares varia de acordo com o estágio de erupção dos segundo e terceiros molares. Karlsson e Bondemark (2006) recomendam que a época favorável para a distalização é 
durante a erupção dos segundos molares??, pois neste momento os efeitos colaterais podem ser minimizados; em contrapartida, Patel et al. (2009) citam que os segundos molares influenciam na inclinação dos primeiros molares. Byloff e Darendeliler (1997) relatam ainda que a presença dos segundos molares aumentam o tempo de tratamento. No entanto existe uma não relação da presença dos segundos molares em relação à inclinação e velocidade de distalização (PATEL et al., 2009).

Kinzinger et al. (2004) ainda afirmam que o impacto da presença ou não dos segundos molares nas distalizações ainda é de muita discussão na literatura, considerando-se que a distalização dos molares superiores é um desafio da terapêutica ortodôntica. Desta forma, como a ortodontia possui uma gama de opções e resultados para os diversos distalizadores intrabucais, cabe ao profissional conhecer a vasta literatura e assim atribuir o distalizador que apresente melhor resultado e menor efeito colateral quando a opção é não utilizar o AEB. Com a amostra estudada, não se pode afirmar qual tratamento obteve maior inclinação, já que não são detectadas diferenças significantes.

\section{CONSIDERAÇÕES FINAIS}

Os resultados em valores mostraram que os aparelhos Jones Jig e Sliding Jig promoveram inclinação distal dos molares, porém o Sliding Jig foi quem apresentou maior inclinação do molar durante o processo de distalização.

\section{REFERENCIAS}

ALMEIDA, R. R.; ALMEIDA, M. R.; INSABRALDE, C. M. B. Um método alternativo de tratamento para a correção da classe II de Angle utilizando o aparelho de Jones Jig. Relato de uma caso clínico. R Dental Press Ortodon Ortop Facial. v.4, n.4, p.37-44, 1999.

ALVES, P. V. M.; BOLOGNESE, A. M.; SOUZA, M. M. G. Movimento distal de molares usando o Sliding-Jig. R Clin Ortodon Dental Press. v.4, n.6, p.83-89, 2006.

ANGLE, EH. Classification of malocclusion. Dental Cosmos. v.1, n.41, p.248-357, 1899.

BOLLA, E; MURATORE, F; CARANO, A; BOWMAN, J. Evaluation of maxillary molar distalization with the Distal Jet: a comparison with other contemporary methods. Angle Ortho. v.72, n.5, p. 481-494, 2002.
BYLOFF, FK; DARENDELILER, A. Distal molar movement using the Pendulum appiliance. Part 1: clinical and radiological evaluation. Angle Ortho. v.67, n.4, p. 249260, 1997.

CAPELOZZA, L. FILHO. Diagnóstico em Ortodontia. Maringá: Dental Press. 2004.

FERREIRA, FV. Ortodontia: Diagnóstico e Planejamento Clínico. 7 ed. São Paulo: Artes Médicas. 2008.

GONÇALVES, MJB; SANTOS, AS; SANTOS, ECA; CAMPANHA, AA; HINO, CT; OLIVEIRA JR, MA. Utilização da ancoragem esquelética na distalização de molares superiores. R Clin Ortodon Dental Press, Maringá. v.8, n.6, p. 89-95, 2010.

HENRIQUES, JFC; PATEL, MP; JANSON, G; FREITAS, MR; CAFFER, DC. Correção das alterações transversais da maxila e da má oclusão de classe II com o aparelho expansor de Haas e distalizador Jones Jig. R Clin Ortodon Dental Press, Maringá. v.4, n.1, p. 73-83, 2005.

HENRIQUES, JFC; NAGEM FILHO, H; HENRIQUES, RP; D'AZEVEDO, MTFS. Tratamento da má oclusão de classe II, $1^{\text {a }}$ divisão, com aparelho Jasper Jamper. Ortho Sci and Prat. v.2, n.6, p. 598-603, 2009.

JANSON, G; BARROS, SEC; SIMÃO, TM; FREITAS, MR. Variáveis relevantes no tratamento da má oclusão de Classe II. R Dental Press Ortodon Ortop Facial, Maringá. v.14, n.4, p.149-157, 2009.

KARLSSON, I; BONDEMARK, L. Intraoral maxillary molar distalization, movement before and after eruption of second molars. Angle Ortho v.76, n.6, p. 923-929, 2006.

KINZINGER, GSM; FRITS, UB; SANDER, FG; DIEDRICH, PR. Efficiency of a pendulum appliance for molar distalization related to second and third molar eruption stage. Am J Orthod Dentofacial Orthop n.125, p.8-23, 2004.

KINZINGER, GSM; EREN, M; DIEDRICH, PR. Treatment effects in intraoral appliances with conventional anchorage designs for non- compliance maxillary molar distalization. A literature review. Eur J Orthod v.30, n.6, p.558-571, 2008.

KLOEHN, SJ. Guiding alveolar growth and eruption of teeth to reduce treatment time and produce a more balanced denture and face. Angle Ortho v.17, n.11-2, p.10-33, 1947.

LEIROS, MLM; PINTO, LP. Estudo comparativo dos efeitos biológicos utilizando-se os aparelhos ortodônticos Jones Jig e Pendulo. R Clin Ortodon Dental Press. Maringá v.4, n.6, p.63-71, 2006.

LUCATO, AS; BOECK, EM; VEDOVELLO, SAS; PEREIRA NETO, JS; MANGNANI, MBBA. Sliding-Jig: confecção e mecanismo de ação. R Clin Ortodon Dental Press, Maringá v. 2, n. 6, p. 10-17, 2004. 
18. MANHÁES, FR; VEDOVELLO FILHO, M; KURAMAE, M; LUCATO, AS; VALDRIGHI, HC. Sistema Ertty para distalização de molares. Relato de casos clínicos. R Clin Ortodon Dental Press. Maringá v.8, n.5, p. 76-88, 2009.

MAIA, JE; OLIVEIRA, AG; OLIVEIRA Jr, G; OLIVEIRA JR, JN; SILVEIRA, CA. Estudo cefalométrico comparativo da inclinação axial mésio-distal dos molares superiores, da altura facial ântero-inferior e ângulo nasolabial após o emprego de dois sistemas de distalização intrabucal: Distal Jet e Jones Jig. J Bras Ortodon Ortop Facial v.9, n.50, p.121-133, 2004.

MALTAGLIATI, LA; MONTES LPA; BASTIA, FMM; BOMMARITO, S. Avaliação da prevalência das seis chaves de oclusão de Andrews, em jovens brasileiros com oclusão normal natural. R Dental Press Ortodon Ortop Facial, Maringá v.11, n. 1, p. 99-106, 2006.

MAVROPOULOS, A; SAYINSU, K; ALLAF, F; KILIARIDIS, S; PAPADOPOLOS, MA; KELES, AO. Noncompliance unilateral maxillary molar distalization: a three-dimensional tooth movement analysis. Angle Ortho, v.76, n.3, p.382-387, 2006.

MOSCARDINI, MS. O “Sliding- Jig” na prática ortodôntica. $\mathbf{R}$ Clin Ortodont Dental Press, Maringá v.9, n.2, p.59-64, 2010.

OLIVEIRA, JMM; ETO, LF. Avaliação radiográfica dos efeitos do aparelho Jones Jig nas distalizações intra-bucais: um estudo piloto. R Dental Press Ortodon Ortop Facial, Maringá v.9, n.5, p. 20-27, 2004.

PATEL, MP; HENRIQUES, JFC; JANSON, G; FREITAS, MR; AALMEIDA, RR. Avaliação cefalométrica das alterações dentoesqueléticas de jovens com má oclusão de classe II dentária tratados com distalizadores Jones Jig. R Dental Press Ortodon e Ortop Facial. Maringá v.14, n.3, p. 83-93, 2009.

PATEL, MP; JANSON, G; HENRIQUES, JFC; ALMEIDA, RR; FREITAS, MR; PINZAN, A; FREITAS, KMS. Comparative distalization effects of Jones Jig and Pendulum appliances. Am J Orthod Dentofacial Orthop v.135, n.3, p. 336-342, 2009.

PAUL, LD; O'BRIEN, KD; MANDALL, NA. Upper removable appliance or Jones Jig for distalizing first molars? A randomized clinical trial. Orthod Craniofac Res v.5, n.4 p.238-242, 2002.

SANTOS, ECA; SILVA FILHO, OG; REIS, PMP; BERTOZ, FA. Distalização dos molares superiores com aparelho Pendex: estudo em modelos de gesso. R Dental Press Ortodon Ortop Facial, Maringá v.11, n.3, p.71-80, 2006.

SANTOS, ECA; SILVA FILHO, OG; REIS, PMP; BEERTOZ, FA. Distalização dos molares superiores com o aparelho Pendex: estudo cefalométrico propectivo. $\mathbf{R}$ Dental Press Ortodon Ortop Facial, Maringá, v.12 n. 4, p. 49-62, 2007.
SILVA FILHO, OG; SANTOS, EC, SILVA, APJ, BERTOZ, APM. Distalização dos molares superiores com o aparelho Pendex unilateral: estudo piloto cefalométrico. R Clin Ortodon Dental Press, Maringá v.5, n2, p.41-50, 2006.

SILVA FILHO, OG; SANTOS, ECA; SILVA, APJL; BERTOZ, APM. Distalização dos molares superiores com aparelho Pendex unilateral: estudo piloto com radiografia panorâmica. R Dental Press Ortodon Ortop Facial, Maringá v.12, n.1, p. 56-66, 2007.

SUGUINO, R; FURQUIM, LZ; RAMOS, AL. O aparelho Jones Jig. R Dental Press Ortodon Ortop Facial, Maringá v.5, n.3, p.83-116, 2000.

THULER, RCSB; CORREA, RCO; FERREIRA, AC; MACEDO, A. Correção da classe II com aparelho estético ortodontia lingual, pendex e mini-implantes. Ortho Sci Prat, v.2, n.7. p.748-752, 2009.

VARGERVIK, K; HARVOLD, EP. Response to activator treatment in Class II malocclusions. Am J Orthod Dentofacial Orthop St. Louis, v.88, n.3, p.242-251, 1985. 